\title{
Análise de Redes Sociais em Blogs de Pessoas com Necessidades Especiais (PNE)
}

\author{
Prof. Dra. Liliana Maria Passerino (UFRGS). E-mail: liliana@ cinted.com.br \\ Prof. Dra. Sandra Portella Montardo (Feevale). E-mail: sandramontardo@feevale.br \\ Arnoldo Benkenstein (BIC CNPq Feevale). E-mail: jrbenk2.0@gmail.com.
}

Resumo: Este estudo aborda os blogs de Pessoas com Necessidades Especiais (PNE) e de seus familiares como ferramentas de inclusão social via Tecnologias de Informação e Comunicação (TIC). Com o levantamento bibliográfico a respeito de redes sociais na web, percebe-se alguns limites quando se deseja entender o desempenho nesse sentido de PNE e de seus familiares nesse suporte. Frente a isso, os softwares existentes de análise de redes sociais na web podem representar satisfatoriamente essas redes? A partir de uma análise comparativa entre os softwares CMap Tools e Ucinet, pretende-se identificar as características ideais de um software a ser desenvolvido que combine resultados de análise quantitativa e qualitativa desse tipo de redes, que são temáticas e que, por vezes, se debatem com a questão da acessibilidade digital.

Palavras-Chave: Blogs; Pessoas com Necessidades Especiais; Análise de redes sociais em blogs.

Title: Social network analysis in disabled people's blogs.

\section{Social Network Analysis in People Disabled's Blogs}

Abstract: This work approaches blogs of disabled people and their relatives as tools of social inclusion through Technologies of Information and Communication (TIC). A bibliographical study on social network on the web shows that there are some obstacles that make difficult to understand the performances of disabled people and their relatives on the web. Thus, can the existent softwares of social network analysis represent satisfactorily such network? Starting from a comparative analysis of the softwares CMap Tools and Ucinet, this work intends to identify ideal features of a software that combine results of quantitative and qualitative analysis of this kind of network.

Key words: Blogs; Disabled people; analysis of social network in blogs.

\section{Introdução}

Este estudo parte da hipótese que a socialização on-line é fator de inclusão social via Tecnologia de Informação e de Comunicação (TIC) de Pessoas com Necessidades Especiais (PNE) e de seus familiares, uma vez que esse recurso pode ser um canal de informação e de afetividade para os parentes e, ao mesmo tempo, tornar-se um espaço promovedor do desenvolvimento sócio-congitivo para $\mathrm{PNE}^{1}$. Esta discussão da relação

\footnotetext{
${ }^{1}$ Estudos desenvolvidos no NIEE/UFRGS desde 1985 comprovam especialmente estes indícios. Estes estudos podem ser consultados no site http://www.niee.ufrgs.br .
} 
entre Inclusão Social (IS) e Inclusão Digital (ID) via Acessibilidade Digital foi amplamente abordada em um estudo anterior (MONTARDO; PASSERINO, 2007).

Nele trouxemos os aportes de Ladeira e Amaral (1999) que conceituam inclusão social como um processo que se prolonga ao longo da vida de um indivíduo e que tem por finalidade a melhoria da qualidade de vida do mesmo. E destacamos a conceituação de Sposati (1996) na qual a inclusão concentra-se na busca pelo acesso a quatro utopias básicas: autonomia de renda, desenvolvimento humano, equidade e qualidade de vida. Para tanto, de acordo com Azevedo e Barros (2004), é necessário uma redistribuição da riqueza social e tecnológica para os cidadãos. Nesse sentido, e de acordo com Warschauer (2006), a inclusão digital é uma faceta da inclusão social e consiste, além de proporcionar o direito de acesso ao mundo digital para o desenvolvimento intelectual, promover espaços para práticas culturais significativas que tornem os participantes letrados digitalmente, ou seja, não apenas com capacidade técnica de atuar no ciberespaço, mas com capacidade de criar e produzir significados e sentidos nele. Embora a IS de PNE e de seus familiares via blogs ainda não tenha sido demonstrada, entendemos que o que se fala nessas redes sociais selecionadas é importante, pelo menos a partir de uma abordagem que busca verificar processos de inclusão. Nesse sentido, observamos que estudos sobre redes sociais na web, especificamente em blogs, raramente levam em conta o que se diz nesse suporte, de modo que certas redes mesmo que se estruturem em torno de um tema específico, não se atém a ele. Diante da evidência de que blogs de familiares de PNE se restringem a um tema específico (postagens e comentários), e da limitação que a falta de acessibilidade digital em blogs e em sistemas de publicação de blogs representa para $\mathrm{PNE}^{2}$, nosso objetivo é apontar para especificidades deste tipo de redes sociais na web e, conseqüentemente, para os limites e as possibilidades dos softwares desenvolvidos até então para as mesmas.

\section{Análise das Redes Sociais: contexto científico}

Segundo Recuero (2005b), a Análise das Redes Sociais (ARS) ocupou-se da metáfora das redes para estudar a sociedade anos antes da proposição da "ciência das redes", baseada na sociometria e na Teoria dos Grafos (Degenne e Forsé, 1999; Scoutt, 2001). Ambas têm por objetivo "o foco no todo, mais do que nas partes e nas interconexões entre essas partes, em uma tentativa de observar os padrões que unem os

\footnotetext{
${ }^{2}$ Consultar o trabalho de (BEZ, R.; MONTARDO, S.P.; PASSERINO, L, 2007) . para os problemas apresentados
} 
elementos dos sistemas. Essas idéias são comuns à Teoria Geral dos Sistemas (Bertalanffy, 1975); e à Cibernética (Wiener, 2000; Ashby, 1970)" (RECUERO, 2005b, internet). Para Wasserman e Faust (1994), uma rede social consiste de elementos ou atores (nós) ligados entre si por laços sociais (arestas).

Pode-se dizer que o marco inicial desta área do conhecimento se deu com a Teoria dos Grafos proposta pelo matemático suíço Leonhard Euler. Segundo Barabási (2003), na busca pela solução do enigma das pontes de Königsberg ${ }^{3}$, em 1736, Euler vislumbrou os grafos como uma coleção de nós conectadas por links. O autor (2003) sugere que os resultados obtidos por Euler ainda sejam válidos, na medida em que evidenciaram que a construção e a estrutura dos grafos ou redes ajuda-nos a entender a complexidade do mundo que nos rodeia.

Já os matemáticos húngaros Paul Erdós e Alfréd Rényi ocuparam-se da questão: como se formam as redes? Erdós e Rényi chegaram à conclusão que as redes se formam aleatóriamente e que cada nó tem a mesma probabilidade de receber links que os demais, sejam os nós pessoas, neurônios ou empresas. Tinha-se, com isso, o Modelo de Redes Igualitárias, a respeito da qual Barabási (2003) afirma que seus autores estavam mais preocupados com a beleza aleatória dos modelos matemáticos do que em propor um modelo capaz de capturar os padrões de associação de elementos das redes existentes.

Ao se perguntar como as pessoas conseguem empregos, o norte-americano Mark Granovetter propõe um dos mais influentes textos sobre redes gerados na Sociologia, conforme Barabási (2003): “A força dos laços fracos”(1973). Relacionando este ponto à teoria clássica da química sobre ao importante papel que pontes de hidrogênio exercem na molécula da água, Granovetter entendeu que são os apenas conhecidos e não os amigos os responsáveis por indicações a novos empregos.

Barabási (2003) percebe na teoria de Granovetter (1974) a visão de uma sociedade que em muito difere da de Erdós e Rényi. Ao invés de uma estrutura aleatória, Granovetter vê a sociedade constituída por pequenos grupos em que todos se conhecem entre si. Trata-se, então, de muitos conjuntos de nós conectados a todos os outros dentro de um cluster (laços fortes) que se ligam a outros clusters por meio de

\footnotetext{
3 "É possível atravessar as pontes de Königsberg passando em cada uma delas apenas uma vez?", perguntavam-se os moradores da cidade. A resposta é não e ,em sua busca, Euler gerou o primeiro grafo, constituídos de nós conectados por arestas.
} 
laços fracos. Portanto, laços fracos representam "nossa ponte para fora do nosso mundo, já que em lugares diferentes freqüentemente as pessoas obtém suas informações a partir de diferentes fontes além dos seus amigos mais próximos" (Barabási, 2003, p. 43) ou, nas palavras de Granovetter (1983), garantem mobilidade social.

Retomando o modelo das redes aleatórias, os estudos de Milgram (Seis Graus de Separação) e a teoria de Granovetter (1973), Duncan Watts e Steven Strogatz propõem a Teoria Mundos Pequenos ao perceberem que uma rede é composta de nós muito conectados e de outros estabelecidos aleatoriamente. A partir do coeficiente de concentração seria possível constatar que a distância média entre todas as pessoas do Planeta seria a de apenas poucos nós (Barabási, 2003).

Ao criticar o aspecto estático das redes entendidas sob o prisma das redes aleatórias, em que os nós permaneceriam imutáveis durante a vida das redes, Barabási (2003) aponta que este modelo não se aplicaria a redes reais, que crescem, como as que se formam na Internet, por exemplo. Observando o desequilíbrio inerente a uma rede e a diferença dos nós entre si em um estudo empírico, Barabási (2003) constatou que os nós mais conectados de uma rede (os mais antigos) probabilisticamente atrairiam mais links do que os menos conectados. Tem-se assim, a Teoria das Redes sem Escalas, em que ricos ficam mais ricos por meio de conexões preferenciais.

Para Hanneman (2001), as redes podem ser consideradas sociocêntricas e egocêntricas. Enquanto as primeiras são estudadas enfatizando-se todos os atores da rede e possíveis sub-redes, as segundas são enfocadas a partir de apenas um ator da rede. Neste segundo caso, tem-se as redes puras, que considera a relação de um ator principal e de atores secundários entre si, e as rede interconectadas, cujo foco também é a relação entre os atores secundários. Quanto a isso, Recuero (2005a) afirma que nas redes sociocêntricas, o foco é a análise estrutural como grupo social em interação, enquanto que no segundo o foco está no papel social que um indivíduo desempenha em função da sua posição na rede.

Os trabalhos que se concentraram numa análise estrutural embasada na Teoria dos Grafos sofreram com o passar do tempo uma "quantificação" dos atributos considerando: granularidade, densidade, distâncias (em termo de número de nós de um caminho) como aspectos fundamentais da rede. Com isso, acredita-se que se deve enfatizar os aspectos qualitativos que só podem ser compreendidos a partir de uma 
análise das relações que se estabelecem, mas que não deixam de levar em conta atributos dos nós dessas redes.

Ao se questionar sobre esta insuficiência da ARS, Recuero (2005b) propõe um modelo de análise de redes sociais na Internet, constituída de três elementos principais: organização, estrutura e dinâmica. A organização se relaciona à interação social em um grupo. Já a estrutura se refere ao resultado das trocas empreendidas em um grupo, em termos de laços sociais e de capital social. Por fim, a dinâmica trata das modificações sofridas por uma rede com o passar do tempo. Nossa análise se concentrará no item estrutura que, por sua vez, pressupõe a análise de laços sociais e de capital social, já que é buscando a sua visualização que os softwares a serem analisados revelaram-se fundamentais para a atual fase de nossa pesquisa.

Segundo Recuero (2005b), laço social tem a ver com interação social e, de acordo com Breiger (1974), pode ser relacional (voluntário) ou associativo (involuntário, referente a pertencimento). Conforme já foi posto, para Granovetter (1974), laços fortes são aqueles que indicam conhecimento e proximidade mútua entre os atores de um mesmo cluster, enquanto que os laços fracos se caracterizam pelo aspecto esparso e difuso das trocas empreendidas em uma relação (Wellman, 1997 citado por RECUERO, 2005b), mas que, entretanto, constituem fonte alternativa de informações e mobilidade dentro da sociedade.

Já o capital social diz respeito às conexões e tem como elemento a reciprocidade e a confiança, afirma Recuero (2005b). Ao comparar os pontos de vista de Putnam (2000) e Bourdieu (1983), a autora conclui que "o capital social constitui-se em um conjunto de recursos de um determinado grupo obtido através da comunhão dos recursos individuais, que pode ser usufruído por todos os membros do grupo, ainda que individualmente, e que está baseado na reciprocidade" (RECUERO, 2005b, internet), sendo que se deve considerar, para tanto, o conteúdo das mensagens.

Para Bertolini e Bravo (2004), citados por Recuero (2005b), existem cinco tipos de capital social: relacional, normativo, cognitivo, confiança no ambiente social e institucional. Além disso, esses tipos podem ser agrupados enquanto capital social de primeiro nível (relações, normas e leis e conhecimento) e de segundo nível (confiança no ambiente social e presença de instituições).

Ainda que de acordo com este modelo, laços sociais e capital social façam parte da estrutura das redes, em trabalhos posteriores da autora, verifica-se amplamente a 
análise de capital social empreendido em redes sociais na web dispostas em diferentes suportes (Orkut, blogs e fotologs). Já os laços sociais não foram contemplados com a mesma força e interesse. Talvez pelo fato de que as redes analisadas terem sido constituídas independente de uma temática específica, mas a partir de vários pontos de afinidade.

No entanto, uma rede egocêntrica interconectada em blogs de PNE e de seus familiares pressupõe, mais do que a análise do conteúdo das postagens e dos comentários para identificação do tipo de capital social empregado, o contexto de sua proposição, ao menos no caso de pretender-se entendê-la sob o ponto de vista da IS via TIC. Sob esta condição, torna-se necessário entender a motivação dos autores dos blogs ao participar de uma rede temática. Feitas as considerações teóricas e alguns questionamentos quanto a ARS, torna-se necessário verificar o desempenho dos softwares destinados a este tipo de análise.

\section{Material e métodos}

No presente artigo limitamos nossa busca e análise de recursos a apenas dois dos inúmeros softwares disponíveis hoje $\left(\mathrm{AGD}^{4}, \mathrm{Agna}^{5}, \mathrm{Netminer}^{6}, \mathrm{Netcopy}^{7}\right)$. São eles o Cmaptools $^{8}$ e UCINET $^{9}$, sendo este último bastante reconhecido por analistas de redes sociais e especialmente desenvolvido para esta função. Já o CmapTools é uma ferramenta desenvolvida inicialmente para a construção de mapas conceituais colaborativos. Sobre o UCINET, há uma considerável literatura à disposição aos possíveis interessados. Como produto desta análise, apontaremos quais características percebidas nestes programas podem ser úteis para composição de um futuro software a ser desenvolvido pelo grupo. Dedicaremos os próximos parágrafos a uma breve descrição dos dois softwares para posterior avaliação.

\subsection{Cmaptools:}

Nosso grupo de pesquisa, atualmente, utiliza o software Cmaptools como instrumento de visualização da rede de blogs de PNE. Grifa-se a palavra visualização, pois este software não possui ferramentas específicas para ARS capazes de isolar variáveis e gerar rotinas baseadas em dados extraídos da rede. Sua adoção foi necessária

\footnotetext{
${ }^{4}$ Possui algoritmos pré-definidos e ferramentas para desenvolvimento de gráficos bidimensionais.

${ }^{5}$ Software livre para sociometria ou análise seqüencial.

${ }^{6}$ Software desenvolvido por Cyram Co., Ltda.

${ }^{7}$ Primeiro software criado para análise de redes sociais.

${ }^{8}$ Disponível em http://cmap.ihmc.us.

${ }^{9}$ Disponível em http://analytictech.com/dowloadaduc6.htm
} 
numa etapa inicial do trabalho em que se buscava uma representação gráfica mais completa na qual fosse possível a representação não só de grafos, mas de gráficos que indicassem também a diferenciação entre links e comentários estabelecendo valores diferentes para cada elemento sendo assim mais coerente com a visão do projeto. Porém, apesar da intuitividade da interface e dos recursos gráficos disponíveis, o trabalho de transposição de dados para este software revelou-se um processo moroso e pouco funcional.

O CMapTools um software destinado à elaboração de mapas conceituais. Criados pelo psicólogo americano Joseph Novak e baseados na teoria da aprendizagem significativa de David Ausubel. Estes mapas são diagramas bidimensionais com a finalidade de representar conceitos e suas respectivas proposições.

O usuário tem à disposição uma interface WYSIWYG bastante intuitiva que adiciona e renomeia elementos através de poucos movimentos do mouse o que torna acessível o aprendizado da ferramenta. Possui muitos recursos de formatação gráfica, como opção de traço e cores, e formatação textual, como definição de estilos de fontes e correção ortográfica.

Como se destina à representação gráfica de conceitos traz ferramentas também para troca de conhecimento. Uma interessante ferramenta dedica a este fim são as Knowledge Soups a partir das quais é possível a criação colaborativa e on-line de mapas conceituais

\subsection{Ucinet:}

Atualmente na versão 6.170, este software, embora tenha por função principal a elaboração e manipulação de matrizes reticulares, pode ser descrito como um pacote de aplicações para ARS, pois quando instalado traz consigo outros três programas que podem ser utilizados de forma independente. São eles dois visualizadores: Netdraw, que visualiza grafos em duas dimensões, e Mage, que mostra grafos em três dimensões. $\mathrm{O}$ terceiro programa é um manipulador de matrizes alternativo e freeware denominado Pajek.

Um dos pontos interessantes do UCINET é a flexibilidade dos formatos de arquivos utilizáveis, tendo como extensão nativa arquivos dataset(\#\#H). Pode-se também importar planilhas do Microsoft Excel e o formato .net utilizado pelo Pajek. 
Embora ofereça um editor de planilhas,podemos manipular matrizes através de texto no formato DL como o do exemplo abaixo:

$d \ln =5$ format $=$ el1
labels embedded
data:
Blog1 Blog2 1
Blog2 Blog1 1
Blog2 Blog3 1
Blog3 Blog2 1
Blog4 Blog1 1
Blog1 Blog4 1

A utilidade desta forma de entrada de dados é mais visível na presença de grandes quantidades de valores.

Já no campo da análise propriamente dita, além das formas de manipulação das matrizes, o software oferece um leque amplo de rotinas e algoritmos que executam desde o isolamento de variáveis até o teste de hipóteses de comportamento. Devido ao grande número de ferramentas disponíveis e o espaço restrito dedicado a esta explanação apenas citamos alguns recursos úteis para uma análise genérica de redes.

\begin{tabular}{|l|l|l|}
\hline Recurso & \multicolumn{1}{|c|}{ Localização } & \multicolumn{1}{c|}{ Descrição } \\
\hline Grau Nodal & Network $>$ Centrality $>$ Degree & Número de laços diretos do nó. \\
\hline Grau de intermediação & $\begin{array}{l}\text { Network }>\text { Centrality>Betweenness }>\text { Nod } \\
\text { es: }\end{array}$ & $\begin{array}{l}\text { Freqüência de nós } \\
\text { geodésicamente próximos. }\end{array}$ \\
\hline Proximidade & Network>Centrality>Closenness & $\begin{array}{l}\text { Indica o quanto o nó se encontra } \\
\text { afastado da rede. }\end{array}$ \\
\hline
\end{tabular}

Tabela 1. Alguns recursos disponíveis no UCINET

Entre os softwares que acompanham a instalação do UCINET estão os seguitnes: Netdraw, Mage e Pajek. A vantagem do visualizador Netdraw é trabalhar diretamente atrelado a dados reticulares, ou seja, tanto os nós quanto seus atributos têm de ter como origem uma matriz. Desta forma o analista pode se fixar na alteração dos dados enquanto o trabalho mecânico de montagem do gráfico da rede é automatizado. Já o Mage gera gráficos tridimensionais a partir de arquivos do UCINET \#\#H. Finalmente, o Pajek $^{10}$ é um software de ARS para Windows. Possui recursos customizáveis através de algoritmos além de boas ferramentas de visualização. Através dele são possíveis operações também presentes no UCINET, como isolar clusters e análise relacional entre grupos.

\footnotetext{
${ }^{10}$ Significa aranha em esloveno.
} 


\section{Resultados e Discussão}

Após comparação de ambos os softwares, uma possível contribuição do Cmaptools pode ser interface amigável que, conseqüentemente, favorece um aprendizado mais amigável para os pesquisadores. Ou seja, ainda que não se preste à ARS, seu ferramental permite o mapeamento de redes, enfatizando-se o tipo de relação entre os nós.

Embora constata-se que o UCINET possua um abrangente ferramental para uma análise webométrica, este ainda não está apto para considerar o conteúdo textual das postagens e comentários. Desconsidera assim o grau de envolvimento e intencionalidade da mensagem resultando novamente num tipo de análise quantitativa que não vai ao encontro do objetivo deste projeto. Ainda assim as medidas e variáveis por ele isoladas são de fundamental importância para uma avaliação quantitativa da rede.

Assim como as teorias que lhe precederam, os softwares para visualização e de ARS privilegiam aspectos quantitativos das mesmas. Por seu turno, revisões das teorias de redes sociais que buscam aprofundar seus aspectos qualitativos tendem a focar na interação (links), deixando em segundo plano os nós dessas redes. Sob o ponto de vista de uma abordagem de inclusão social via TIC, é importante considerarmos as duas instâncias para uma compreensão mais ampla do fenômeno, considerando-se as redes de PNE e de seus familiares como, quando do seu mapeamento, sociocêntricas e, no momento de sua análise, em egocêntricas interconectadas (Hanneman, 2001).

Além disso, essas redes, que costumam ser temáticas (postagens e comentários se restringem totalmente a questões referentes à Necessidade Especial considerada) e que enfrentam constrangimentos tecnológicos que condicionam a sua existência (falta de acessibilidade digital em blogs e sistemas de publicação de blogs [Bez, Montardo, Passerino, 2007]) requerem: 1) consideração de intenção comunicativa dos autores de blogs e, simultantemente, a identificação da necessidade especial em questão e sua relação com acessibilidade digital; 2) uma combinação entre os tipos de capital social empregados na relação com a idéia de laços fracos e fortes, segundo Granovetter (1973).

\section{Conclusões}


Por um lado, a ARS em blogs de PNE e de seus familiares requer que se leve em conta o tema abordado em suas redes, já que são constituídas com essa finalidade. Com isso, deve-se não só levar em conta o capital social empregado nas trocas sociais empreendidas nessas redes, mas classificar os laços que se formam nelas em fracos e fortes (Granovetter, 1974, 1983), classificando os diferentes tipos de links (blogroll, de postagens, de comentários e do trackback) que conectam os nós dessas redes como laços fracos ou fortes.

Ainda que seja necessária a investigação de outros softwares de ARS, fica claro que o tratamento lingüístico das postagens e comentários dessas redes temáticas, via Processamento de Linguagem Natural (PLN), é imprescindível em um software a ser desenvolvido $^{11}$. A proposta é investigar o desenvolvimento de mecanismos inteligentes para descoberta de conhecimento em blogs que estejam relacionados a temas ligados às diferentes Necessidades Especiais. Por meio de técnicas de PLN, ontologias e um Robô Virtual, esse conhecimento será reunido, organizado e utilizado de forma automática e semi-automática na busca de informações dispersas nos blogs.

\section{Referências}

AZEVEDO, P. H.; BARROS, J. F. O nível de participação do Estado na gestão do esporte brasileiro como fator de inclusão social de pessoas portadoras de deficiência. Revista Brasileira de Ci e Movimento. Brasília, v. 12, n. 1, p. 77-84. Jan/Mar 2004.

BARABÁSI, A-L. Linked. How everything is connected to everything else and what it means for Business, Science, and everyday life.Cambridge: Plume, 2003.

BATAGELJ, V; MRVAR, A. Pajek. Analysis and visualization of large networks. In: PreprintSeries,V.41,2003.Disponívelem:http://www.ijp.si/ftp/pub/preprints/ps/2003/pp 871.pdf. Acesso em 28 out.2007.

BEZ, R.; MONTARDO, S.P.; PASSERINO, L. M. Acessibilidade digital em sites de publicação de blogs e em blogs: limites e possibilidades para socialização on-line de Pessoas com Necessidades Especiais (PNE). In: Anais. XXX Congresso Nacional de Ciências da Comunicação. Santos, SP, 2007. Disponível em cd-rom.

FAZITO, D. A Análise de Redes Sociais (ARS) e a Migração: mito e realidade. Trabalho apresentado no XII Encontro da Associação Brasileira de Estudos Populacionais. Ouro Preto, MG, 4-8 nov. 2002. Disponível em http://www.abep.nepo.unicamp.br/docs/anais/pdf/2002/GT_MIG_ST1_Fazito_texto.pdf . Acesso em 13 out. 2007.

\footnotetext{
${ }^{11}$ Um bolsista Fapergs do Curso de Sistemas da Informação, do Centro Universitário Feevale, será o responsável pelo desenvolvimento de um software neste sentido.
} 
GRANOVETTER, M. The strenght of weak ties. In: American Journal of Sociology, 78, p. 1360-1380, 1973.

. The strenght of weak tis: a network theory revisited. In: Sociological Theory, Volume 1. 1983, p.201-233.

HANNEMAN, R. A. Introduction to Social Network Methods. Riverside: University of California, 2001. Disponível em http://faculty.ucr.edu/ hanneman/SOC157/NETTEXT.PDF. Acesso em 27 out. 2007. LADEIRA, F.; AMARAL, I. A educação de alunos com multideficiência nas Escolas de Ensino Regular. Coleção Apoios Educativos. Lisboa: Ministério da Educação.Departamento da Educação Básica, 1999.

MONTARDO, S. P. PASSERINO, L. Inclusão social via acessibilidade digital: proposta de inclusão digital para Pessoas com Necessidades Especiais (PNE). In: eCompós.Edição8.Abr.2007.Disponívelem:http://www.compos.org.br/ecompos/adm/doc umentos/ecompos08_abril2007_passerino_montardo.pdf

RECUERO, R. Redes Sociais na Internet: considerações iniciais. In: eCompós, v.2, Abr. 2005. (2005a) Acesso em 26 de outubro de 2007.

Comunidades virtuais em redes sociais: uma proposta de estudo In: eCompós, v.4, Dez 2005. (2005b). Acesso em 26 de outubro de 2007.

REVISTA Redes.Disponívelem http://revistaredes.rediris.es/webredes/recursis.htm\#Programas\%20ARS. Acesso em 21 out. 2007.

SILVEIRA, S. A. Inclusão digital, sofware livre e globalização contra-hegemônica. Disponível em http://www.softwarelivre.gov.br/softwarelivre/artigos/artigo_02. Acesso em 4 dez. 2006.

SCHLÜNZEN, E. T. M. A tecnologia como inclusão de Pessoas com Necessidades Especiais (PNE). In: PELLANDA, N. et al. Inclusão digital: tecendo redes afetivas/cognitivas. Rio de Janeiro: DP\&A, 2005. pp. 195-210.

SPOSATI, A.A fluidez da inclusão/exclusão social. Ciência e Cultura, vol. 58, no. 4., São Paulo, Out/Dec. 2006. 1996.

Mapa da Exclusão/Inclusão da Cidade de São Paulo. São Paulo: PUC-SP,

WARSCHAUER, M. Tecnologia e Inclusão Social. A exclusão digital em debate.

São Paulo: Senac, 2006.

WASSERMAN, S.; FAUST, K. Social network analysis: methods and applications. Cambridge: Cambridge, 1994.

WATTS, D. Six degrees. The science of a connected age. New York: W. W. Norton \& Company, 2003. 